\title{
Feasibility of a Wireless Health Monitoring System for Prevention and Health Assessment of Elderly People
}

\author{
Viveca Jiménez-Mixco, María F. Cabrera-Umpiérrez, \\ Maria Panou, Matthias Struck, Silvio Bonfiglio
}

\begin{abstract}
The work presented in this paper comprises the methodology and results of a pilot study on the feasibility of a wireless health monitoring system designed under main EU challenges for the promotion of healthy and active ageing. The system is focused on health assessment, prevention and lifestyle promotion of elderly people. Over a hundred participants including elderly users and caregivers tested the system in four pilot sites across Europe. Tests covered several scenarios in senior centers and real home environments, including performance and usability assessment. Results indicated strong satisfactoriness on usability, usefulness and user friendliness, and the acceptable level of reliability obtained supports future investigation on the same direction for further improvement and transfer of conclusions to the real world in the healthcare delivery.
\end{abstract}

\section{INTRODUCTION}

It is remarkable how healthcare systems across Europe are facing serious problems to cover their costs. The rationale for that does not only rely on the methods for raising funds, which are inadequate, but, of even greater concern, the costs themselves are rising. Some of the main factors of increasing healthcare costs in Europe are the ageing populations, the costly technological advances, and patient demand driven by less healthy lifestyles. The European Innovation Partnership on Active and Healthy Ageing, launched in 2011, pursues a triple challenge for the future of Healthcare in Europe: a) fostering healthy, active and independent EU ageing citizens b) improving the sustainability and efficiency of social and health care systems, and c) boosting and improving the competitiveness of the markets for innovative products and services [1]. Based on these, the future of healthcare will be leaded by more effective preventive measures and fundamental lifestyle changes, as well as patients taking more responsibility for their own health, treatment and care [2]. Therefore, in 2030 Europe healthcare delivery scenario could be designed over an e-health scenario focused on the most vulnerable population, where preventive medicine would prevail over treating the sick. For that purpose, it is essential that the discussion focuses on the most promising approaches to maximize the health and wellbeing of Europe's population [3][4]. In this direction, the European Union is investing thoroughly for the research and development of new healthcare technologies to help empower the patients and particularly the older adults so that they become able to personally take care of their health status and prevent further illness or accidents. The work presented in this paper has been carried out within the framework of OASIS European project [5][6]. It comprises the methodology and evaluation results of the Oasis Health Monitoring System (OHMS), a personalized e-health solution based on wireless technologies designed as an effort to advance in a new generation of healthcare services for the elderly. It is devoted to -going beyond the monitoring of the health status- providing them with an easy-to-use system to personally manage their health status and assuring their well-being by means of a constant remote control by professional caregivers. Tests were performed under different scenarios (i.e. lab tests and real home unsupervised environments) in four countries across Europe (Romania, Bulgaria, Germany and Italy), involving over a hundred of users.

\section{ThE OASIS HEALTH MONITORING SySTEM}

OASIS project's main objective was to explore the potential of Information and Communication Technologies in all aspects of daily life of the elderly to create ambient assisted living environments that supported them to meet their social, emotional and psychological needs, take care for their long-term conditions, and prevent further illnesses or accidents. With that aim, an innovative, modular, holistic and easy to use reference architecture was implemented, facilitating interoperability, seamless connectivity and sharing of content between different services in all application domains relevant to applications for the elderly. One of these applications is the OHMS, which facilitates health profile management and remote monitoring of user's biomedical parameters, motivates the user to follow a healthier lifestyle and trigger alerts in case of emergency events or messages from the doctor. Being integrated in the aforementioned architecture, the main innovation of the OHMS compared to other healthcare systems is that, a) on one hand the system is able to exchange useful information to other domains (e.g. nutrition, environment, brain skills, transport, etc.) with any other application of the platform through the user's profile, giving the health professional enriched data about the context of the user, and b) on the other hand, external healthcare providers will be able to integrate their services in the platform and connect them to the OHMS with a very small effort [7]. The main components of the system are the following: 


\section{A. Sensors}

Biomedical and activity data directly related with the health status maintenance are obtained from six wireless biomedical sensors that are used to perform continuous monitoring of the elderly health status (i.e. SpO2, Glucose, Blood pressure, ECG, Weight, Activity Level).

\section{B. User's terminal}

The application is available on a tablet PC for the elderly user. The intelligence allocated in the terminal is based on four main modules: Health profile definition and personalization (relevant information of the user, mainly related to his habits and health status), Remote monitoring through sensors (assessment of health and activity parameters), Health coaching for prevention through information, education and motivation (including the possibility of contacting the physician through the application), and Alerting and assisting (panic button and emergency events detection) [7].

\section{User's terminal}

The health applications server stores the health assistance applications that allow elderly users to receive the health contents and advices from the health coach module on their terminals, and caregivers/control centers to control the elders' health status and be warned about emergency situations.

\section{Health professional's terminal}

The health professional is provided with the Medical Center desktop application, which allows him to perform a continuous follow-up of the user's health status, as well as to be proactive and make decisions on the performance of the user's application.

\section{EVALUATION METHODOLOGY}

This section gives the details of the evaluation methodology that was further applied to a large-scale, realworld problem with variable arm sets to validate the feasibility of the proposed system.

\section{A. Users sample and Pilot sites}

Define Participants aged 55+ years who met the criteria for healthy ageing were recruited through key organizations such as senior occupational centers, sheltered homes, user associations and primary care centers. By healthy ageing we consider the segment of elderly population that are healthy, but may be experiencing mild cognitive and physical deterioration due to age. Table I depicts the number of users recruited per pilot site. Four countries across Europe were selected for the evaluation, Romania, Germany, Bulgaria and Italy, taking advantage of instrumentation and knowledge of each specific site. This selection was based on a number of factors, mainly to represent different geographic regions of Europe and potentially all test environments per site: living labs for technical verification and iterative development, sheltered homes for assisted living and user communities-related, and real home environments, namely private homes, for real world applications. The approach intended to obtain valuable feedback from the contrast of the different environments and scenarios and thus to support the validity of findings for the potential transfer of results to the real world. Finally, in each pilot site, scenarios with interactive steps were applied based on specific use cases for each application developed.

TABLE I. USERS AND PILOT SITES

\begin{tabular}{|c|c|c|}
\hline Pilot Site & $\begin{array}{c}\text { No of elderly users } \\
\text { (female/male), Location }\end{array}$ & $\begin{array}{c}\text { No of } \\
\text { caregivers }\end{array}$ \\
\hline Romania & $\begin{array}{l}8(4 M, 4 F) \text { home } \\
\text { environments } \\
\text { (apartments/houses) }\end{array}$ & - \\
\hline Germany & $\begin{array}{l}70 \text { ( } 31 \mathrm{~F}, 39 \mathrm{M}) \text {, of which } 11 \\
\text { at sheltered homes (however, } \\
55(31 \mathrm{~F}, 24 \mathrm{M}) \text { users are } \\
\text { included in the analysis since } \\
\text { they provided a full set of } \\
\text { questionnaires) field strength }\end{array}$ & - \\
\hline Bulgaria & $\begin{array}{l}19 \text { ( } 14 \mathrm{~F}, 5 \mathrm{M}) 15 \text { participants } \\
\text { were living in sheltered } \\
\text { homes and four were living } \\
\text { independently. }\end{array}$ & $\begin{array}{l}10 \text { professional } \\
\text { caregivers }\end{array}$ \\
\hline Italy & $\begin{array}{l}34(14 \mathrm{~F}, 20 \mathrm{M}) \text { in user formm } \\
\text { and big senior centers }\end{array}$ & $\begin{array}{l}15 \text { senior center } \\
\text { managers }\end{array}$ \\
\hline
\end{tabular}

\section{B. Assessment criteria and Evaluation tools}

The assessment criteria for the evaluation were defined according two main categories: a) technical evaluation: reliability and b) user acceptance evaluation: usability, user friendliness, medical privacy, daily life improvement and emergency intervention improvement. The evaluation was organized mainly on an individual basis. Each participant was provided with the corresponding equipment and assigned a person who played the role of caregiver. The equipment provided to each user depended on the test environment and pilot sites ethical requirements (sensors without a CE label could not be tested in all scenarios). Prior to trying the healthcare services offered by the application, users were referred either to a readable/viewable training module or a live demonstration by the pilot site responsible evaluator. Then, participants were given enough time to get familiar with this application and its technical capabilities. The aim of the evaluation was to test the health monitoring application in an unsupervised environment. In order to achieve this goal, the elderly were asked to use the application at home during a week and with the collaboration of a caregiver. Data were acquired according to a predefined protocol that was supervised by a clinician. After the testing period that ranged from three weeks to one month, the results were gathered through a self-evaluation questionnaire and a set of surveys. Also, focus groups were conducted in order to share experiences, comments, opinions, gather important feedback on the acceptability of the application and, most importantly, to gain insight that was not possible to obtain by close-ended, and therefore, constraining questionnaires. The study was approved by the appropriate ethics committees. 


\section{RESUlTS}

\section{A. Performance by elderly users}

The majority of participants (78\%) were able to complete the tasks they were assigned to during testing phase always, or most of the time. Some of the participants encountered problems while trying to complete the requested task due to external factors such as loss of internet connection. Individual malfunction occasions (also due to internet loss) were similar across sites. Almost two thirds of users mentioned (65\%) that the system performance was very fast or fast. In addition, almost all participants (92\%) stated that the information received was correct and $63 \%$ of users responded that the information received by the system was the information requested. Users across pilot sites were highly satisfied ( $88 \%$ ) from the overall services offered they believed that it met their needs. Therefore, the system covered the needs of most participants. This is an important finding for not only investigating the viability of the system but, also, measuring the success of developing a system for those who will use it in the future.

\section{B. Perfomance assessment by caregivers}

Caregivers indirectly assessed the performance and usability of the system by evaluating how supportive -with regards to their everyday tasks and duties -the offered services were. Overall, most users accessed the system with a PC $(36.4 \%)$ or tablet PC (36.4\%). In general, users 'occasionally' asked caregivers for help while using the system with the allocated device $(45.5 \%)$ followed by 'sometimes' (30.3\%). Most caregivers reported that they were not so sure if the use of the system decreased their workload with regards to taking care the elderly or carrying out related tasks $(43.6 \%)$, compared to $35.9 \%$ who were confident that some workload was reduced because of the system

\section{Usability assessment by elderly users}

The majority of users (84\%) reported high usefulness for the application. The same holds true for all participating sites. Two thirds of users $(66.7 \%)$ stated that the health monitoring application is very reliable. Similar to overall usefulness ratings, users from different country sites share the same positive view about the reliability of this application. There was no concern about privacy loss or leaks in all sites and, of course, overall (81.3\%).

Users trusted the health monitoring application and believed that their personal data (e.g. profile, personal health information, doctor appointments, emergency events) were safe with the application. This is a key finding for the safety and soundness of the system as health-related data are very sensitive. Overall, participants believed that this application would improve users' health and wellbeing $(86.2 \%)$. Nevertheless, differences were found among the pilot sites on this respect. The users from the Romanian pilot site stated that this application would not improve their overall health status. On the contrary, users from both the German and Bulgarian pilot sites reported positive scores about the potential impact of this application to their health. Reviewing their health profile took the highest priority as the most useful function in all sites followed by use of medication manager and health alerts, as shown in Figure1. (Note that Fig. 1 and 2 don't include the Italian pilot. This is because the data reported from this site referred to the entire application, not to single modules):

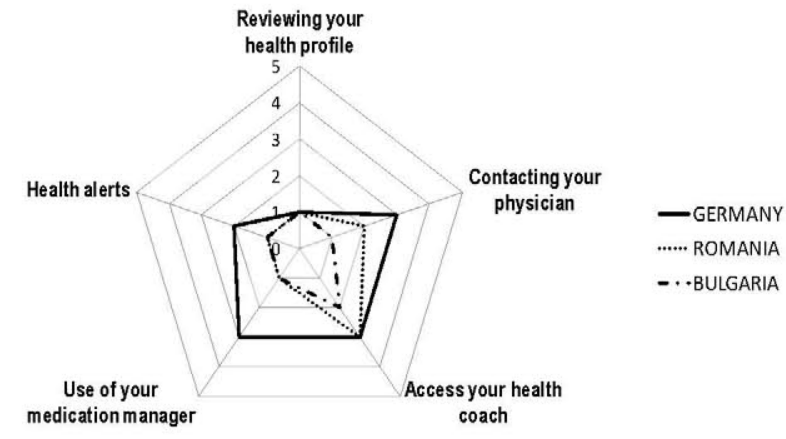

Figure 1. Median usefulness ratings of Health Monitoring Functions (1: most useful tool to 5: least useful tool).

Contacting the physician and accessing health coaching exercises were ranked as second or third priority as added functions. Even though health coach was not prioritized as an essential function for the health monitoring application, many users $(68.8 \%)$ are willing to use this tool in the future. This choice is closely followed by the tool for reviewing your health profile $(60.5 \%)$ and health alerts option $(59.8 \%)$ As these findings are heavily influenced by the number of participants per site, it is important to look closely at the differences among sites. According to the graph below, willingness-to-use specific tools in the future differs per pilot site.

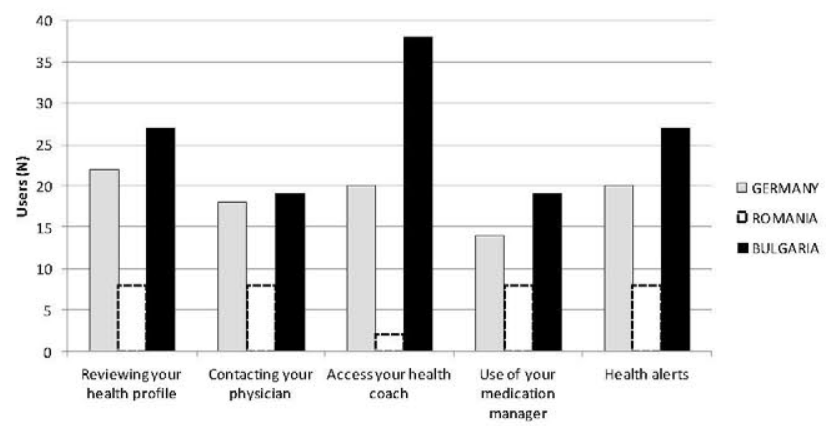

Figure 2. Willingness to use specific OASIS tool after the project end

Users at the German site showed slight preference to the health profile reviewing tool, but all the rest followed closely this specific tool. Users from the Bulgarian site showed clear preference for the health coach access tool, just the opposite to what Romanian users rated. These findings emphasize the need for a flexible, adaptable, and customizable healthcare system.

\section{Usability assessment by caregivers}

The Caregivers participated directly the tests in the Bulgarian and Italian pilot sites and, in general, (84\%) they reported high usefulness for the health monitoring application. This was, also, the case for the individual pilot 
sites. In the same way, caregivers $(90 \%)$ were not concerned for any arising privacy issues. This finding was common in the pilot sites, as well. The majority of caregivers $(85 \%)$ stated that this application would improve the wellbeing of elder users. However, there were discrepancies between the two pilot sites. In the Bulgarian pilot site, users were absolutely sure that this application would improve users' health but in the Italian pilot site most users were neutral. Overall, and for each pilot site, the most useful function was reported to be the creation of a health profile. Table II presents the success rates appointed for the application, giving an overall, mean score above $70 \%$ that was the initial target set within the project. Based on the results of the large-scale evaluations realized in the four pilot countries, we can deduce that the system was perceived as useful with limited to none reliability issues.

TABLE II

KEY PARAMETERS ASSESSMENT RESULTS

\begin{tabular}{ll}
\hline \hline Parameter & Result (\%) \\
\hline Usability & 82 \\
Reliability & 66.7 \\
User friendliness & 81.5 \\
Usefulness & 84
\end{tabular}

Users and caregivers were not concerned about data protection and were positive about the significant contribution of the system to their overall wellbeing and independent living. High levels of usability were rated: the system is easy to start, easy to learn, and easy to use. The layout and color contrast was clear, text was readable and information was easy to locate on the screen. The overall performance was very good and the system's reactions were fast. Occasional crashes and internet connections were mentioned but they were mostly related to the devices rather than to the system itself. It is evident across all findings discussed that overall usefulness, reliability, usability, and user-friendliness were in most occasions above $80 \%$. Also, the positively rated equipment, service cost and quality of service, all key factors, determine the future marketability of the proposed system.

\section{CONCLUSION}

Eventually, the healthcare system developed within the framework of OASIS project has been tested iteratively and thoroughly by over a hundred of end users (in North, Central, South and East Europe) and their caregivers in 2011, providing high acceptability scores. This paper presented the methodology and consolidated results of the process. The contrast of environments, cultural factors and methodological perspectives obtained from the different pilot sites definitely enhanced the results, supporting the validity of findings and the potential for transferring conclusions to a real cost-effective healthcare scenario in the area of independent living of the ageing population. Findings outlined a promising future for the system in both familiar and unfamiliar locations. It could contribute towards enhanced confidence and independence. Reliability, equipment, service cost and quality of service were all key factors determining the future marketability of the concept. The future objective is a wide deployment of the system within a large scale pilot that will involve relevant stakeholders such as public authorities, healthcare providers and users associations. The results will gather evidence of the socio-economic potential of new integrated healthcare models.

\section{ACKNOWLEDGMENT}

This work has been carried out as part of OASIS project, co-funded by the European Commission under the Seventh Framework Programme (FP7, ICT-2007-215754). We would like to thank the whole OASIS consortium for its valuable contribution to this work. We are grateful to the pilot site managers for their strong support and dedication.

\section{REFERENCES}

[1] "Active Senior Citizens for Europe: A Guide to the EU". Report from the AGE Platform Europe. November, 2011.

[2] "The future of healthcare in Europe". Report from the Economist Intelligence Unit Limited 2011. Sponsored by Janssen pharmaceutical company.

[3] Jun Yan; Chao Chen; Xiaohui Duan; Anpeng Huang; Zhinan Li; , "A Wireless Healthcare System Based on Mobile Internet Platform," Information Engineering and Computer Science (ICIECS), 2010 2nd International Conference on , vol., no., pp.1-4, 25-26 Dec. 2010

[4] K. Leichsenring, "Integrated care for older people in Europe-latest trends and perceptions". International Journal of Integrated Care, Volume 12,30 January 2012.

[5] Bekiaris, E. and Bonfiglio, S. "The OASIS Concept". Universal Access in HCI, Part I, HCII 2009, Lecture Notes in Computer Science 5614, 2009. ISBN: 978-3-642-02706-2.

[6] OASIS project Website. Online: www.oasis-project.eu.

[7] Jimenez-Mixco,V. et al. "Personalized e-Health for Elderly Self-care and Empowerment". Advances in Intelligent and Soft Computing vol 153, p213-216 (Springer). ISBN: 978-3-642-28782-4

[8] Y. Ren et al. "Monitoring patients via a secure and mobile healthcare system," Wireless Communications, IEEE, vol.17, no.1, pp.59-65, February 2010.

[9] D. Maltby et al. "Checklist for Preparing a Validation Plan: Updated Version". Converge project. 1998.

[10] R.G. Fichman et.al. "The Role of Information Systems in Healthcare: Current Research and Future Trends". Information Systems Research, Vol. 22, No. 3, September 2011, pp. 419-428.

[11] "Demographic change and work in Europe". European Foundation for the Improvement of Living and Working Conditions, 2010.

[12] Patient J. Clemensen and J. Rasmussen. Empowerment and New Citizen Roles through Telehealth Technologies. eTELEMED 2011: Third International Conference on ehealth, Telemedicine and Social Medicine.

[13] Beyette, F.R. et al. "Point-of-Care Technologies for Health Care", IEEE Transactions on Biomedical Engineering, Vol. 58, pp. 732 735, March 2011.

[14] José Luis Monteagudo Peña and Oscar Moreno Gil. "e-Health for Patient empowerment in Europe". Informes, Estudios e investigación 2007. Ministerio de Sanidad y Consumo, Instituto de Salud Carlos III 